\title{
Isolation and Characterization of Marinobacter sp. KS-1, which Produces a Chondroitin Sulfate-like Mucopolysaccharide
}

\author{
Dae-Sung Lee ${ }^{1}$, Kyung-Suk Kim², Myung-Suk Lee ${ }^{3}$ and Young-Mog Kim ${ }^{4 *}$ \\ ${ }^{1}$ POSTECH Ocean Science and Technology Institute, Pohang University of Science and \\ Technology (POSTECH), Pohang 790-784, Korea \\ ${ }^{2}$ Korea Advanced Food Research Institute (Busan Branch), Busan 613-816, Korea \\ ${ }^{3}$ Department of Microbiology, Pukyong National University, \\ Busan 608-737, Korea \\ ${ }^{4}$ Department of Food Science and Technology, Pukyong National University, \\ Busan 608-737, Korea
}

\begin{abstract}
In an effort to isolate a bacterium producing chondroitin sulfate (CS), a marine bacterium, KS-1, which produces mucopolysaccharides, was isolated from seawater and identified as Marinobacter sp. based on analyses of its morphological and biochemical traits and 16S rDNA sequence. Agarose-gel electrophoresis showed that the KS-1 strain produces a CS-like mucopolysaccharide. Structural analysis using Fourier transform infrared spectroscopy revealed that the structure of the CS-like mucopolysaccharide produced by Marinobacter sp. KS-1 is similar to that of dermatan sulfate (CS B). However, the molecular mass of the CS-like mucopolysaccharide is higher than that of standard chondroitin sulfates. Considering the above results, we conclude that the Marinobacter sp. KS-1 produces a CS-like mucopolysaccharide that differs somewhat from CS B in molecular mass.
\end{abstract}

Key words: Chondroitin sulfate, Marine bacterium, Marinobacter sp., Mucopolysaccharide

\section{Introduction}

Chondroitin sulfates (CSs) are a type of mucopolysaccharide that consist of D-glucuronic acid, $\mathrm{N}$-acetyl-D-galactosamine, and a sulfate group. Depending on the location of the sulfate group in the basic skeleton of the disaccharide, the molecules are classified as chondroitin 4-sulfate (CS A), dermatan sulfate (CS B), and chondroitin 6-sulfate (CS C) (Kang et al., 1994). CSs are known to be effective in the control of cell electrolytes (Tanaka, 1978), the protection of articular tissue and the formation of bone by calcification, the suppression and prevention of atherosclerosis with blood clarification (Prince and Navia, 1983), corneal protection (Mac-Rae et al., 1983), the suppression of bacterial infections (Smith and Schurman, 1983), anticoagulation of blood (Bjornsson et al., 1982; Mourao et al., 1996), and anti-inflammatory and pain relief effects (Fontenele et al., 1996). In addition, CSs are also effective

*Corresponding author: ymkim@pknu.ac.kr against cancer (Moon et al., 1998), asthma, miasma, thirst, trench mouth, and laryngitis (Hjerpe et al., 1979; Krueger et al., 1992; Ha and Kim, 1999). Currently, as the physiological activities of CSs are in the spotlight, many studies are being conducted on CSs for use in medical products, cosmetics, and functional food materials. The domestic consumption of CS products relies heavily on imports, and the amount of imports is increasing every year.

Many suppliers obtain CSs commercially from mammalian species, which are more cost-effective (Im et al., 2009). However, the frequent occurrence of animal epidemics, such as mad-cow disease, foot and mouth disease, and hog cholera, has been a serious issue that mitigates the use of CSs from mammalian sources (Sim et al., 2005; Im et al., 2009; Kim et al., 2010). Thus, CSs are currently purified from marine sources (Im et al., 2009). Among them, shark cartilage is the one most commonly used as a commercial source (Im et al., 2009; Kim et al., 2010). However, several problems are likely to occur with this source, such as overfishing, rising prices of this raw material, and environmental pollution caused by 
the dumping of unused shark parts into the ocean after fishing. Therefore, other organisms need to be investigated as alternative sources of CS production. The objective of this study was to evaluate the possibility of CS production using a microorganism.

\section{Materials and Methods}

Screen for a mucopolysaccharide-producing microorganism

To isolate a bacterium producing CSs, seawater was collected from the Haeundae Coast, Korea, and the samples were smeared on PPES-II agar medium (Taga, $1968 ; 0.2 \%$ polypeptone, $0.1 \%$ proteose peptone, $0.1 \%$ yeast extract, $0.1 \%$ soytone, $0.001 \%$ ferric citrate, and $2.5 \% \mathrm{NaCl}$ ). After incubation at $25^{\circ} \mathrm{C}$ for 5 days, each bacterial colony on the plate was cultured for $48 \mathrm{~h}$ at $25^{\circ} \mathrm{C}$ in PPES-II broth medium. A screen for CS-producing bacteria was carried out as described by Tandavanitj et al. (1989). The bacterial cells were harvested by centrifugation $(7,000 \times g, 30 \mathrm{~min})$ and suspended in $1 \%$ phenol solution. Two volumes of ethanol were then added after removing the supernatant by centrifugation $(30,000 \times g, 2 \mathrm{~h})$. The polysaccharide strands formed by the previous treatment were twined around a glass rod and dissolved in water. The solution was subjected to the carbazole-sulfuric acid test, and bacteria that caused a dark red color in the test were selected as candidate strains producing a mucopolysaccharide.

\section{Identification of a mucopolysaccharide-producing microorganism}

The strains isolated in this study were identified by morphological observations, conventional biochemical tests, and 16S rDNA sequence analysis. Morphological observations and conventional biochemical tests were performed using methods described by Smibert and Krieg (1994). Analysis of the $16 \mathrm{~S}$ rDNA sequence was carried out based on Moyer et al. (1994). Polymerase chain reaction (PCR) was performed using intact cells treated for $5 \mathrm{~min}$ at $95^{\circ} \mathrm{C}$ as a template. The thermal profile used was 35 cycles of denaturation for $30 \mathrm{~s}$ at $94^{\circ} \mathrm{C}$, annealing for $30 \mathrm{~s}$ at $55^{\circ} \mathrm{C}$, and extension for $1 \mathrm{~min}$ at $72^{\circ} \mathrm{C}$. A final extension step consisting of $5 \mathrm{~min}$ at $72^{\circ} \mathrm{C}$ was included. The $16 \mathrm{~S}$ rDNA amplified by PCR was purified, inserted into the pGEM-T Easy vector (Promega, Madison, WI, USA), and sequenced. The resulting sequence data were analyzed using
BLASTN (http://www.ncbi.nlm.nih.gov/BLAST). The sequences were aligned using the program CLUSTAL W, and a phylogenetic tree was generated using the MEGA 2.0 program.

\section{Agarose-gel electrophoresis}

According to the method of Volpi (1999), mucopolysaccharides produced by the isolated strain were further separated by agarose-gel electrophoresis. Agarose gels were made as $1 \%$ agarose in $0.04 \mathrm{M}$ barium acetate buffer ( $\mathrm{pH}$ 5.8). Using $0.05 \mathrm{M} \mathrm{1,2-}$ diaminopropane ( $\mathrm{pH} 9.0$ in the form of acetic acid) as the tank buffer, the gel was electrophoresed for 80 $\mathrm{min}$ at $50 \mathrm{~mA}$. After electrophoresis, the gel was dyed for at least $6 \mathrm{~h}$ in $0.1 \%$ cetyltrimethylammonium bromide. The solution was observed in a dark background and dyed again in toluidine blue (2\% in 50:49:1 ethanol:water:acetic acid) for 15-30 min. The gel was then decolored using a decoloring agent (50:49:1 ethanol:water:acetic acid) to verify the existence of mucopolysaccharides.

\section{Fourier transform infrared spectroscopy (FT-IR) analysis}

To verify the structure of the CS extract, a chemical structural analysis was conducted using an FT-IR5300 spectrophotometer (JASCO; Oklahoma, USA) at the Cooperative Laboratory Center, Pukyong National University.

\section{CS content}

The CS content was tested according to the method of Tandavanitj et al. (1989). The harvested cell pellets (150 mg of wet weight) were mixed with water to $50 \mathrm{ml}$. The solution $(10 \mathrm{ml})$ was then passed through filter paper (Advantec 5A; Toyo, Tokyo, Japan) and used as a test solution. Five milliliters of borax sulfate solution was placed in Nessler tubes and cooled with ice water. One milliliter each of the sample and standard solutions was carefully added and cooled with mixing. After 10 min of heating in a water bath, the solutions were immediately cooled using ice water. To each solution, $0.2 \mathrm{ml}$ of carbazole was added and mixed. After 15 min of heating in a water bath, the solutions were cooled to room temperature using ice water. A water solution was prepared as a control using the same procedure. The absorption of each sample at $530 \mathrm{~nm}$ was measured, and the mucopolysaccharide content was calculated according to the method of Tandavanitj et al. (1989).

\section{Results and Discussion}


Isolation and identification of a mucopolysaccharide-producing microorganism

Mucopolysaccharides, including CSs and heparan sulfate, were obtained commercially from marine sources such as whale shark, skate, squid, salmon, king crab, and sea cucumber (Im et al., 2009). Among these, shark cartilage is the most commonly used commercial source (Im et al., 2009), but overfishing and rising prices of this raw material will likely occur in the future. Therefore, other organisms need to be developed as alternatives to replace this marine resource.

We attempted to isolate a microorganism that produces CS. In the current study, we isolated a total of 150 colonies with distinguishable shapes from seawater samples. Among them, one species, KS-1, was selected as a candidate strain producing mucopolysaccharide as revealed by the test of Tandavanitj et al. (1989). To identify this strain, morphological, biochemical, and genetic analyses were performed. Gram staining revealed that this strain is Gram negative (data not shown). The results of biological and physiological tests are listed in Table 1. For the genetic characterization of KS-1, PCR was carried out to amplify the $16 \mathrm{~S}$ rDNA as described above. The 16S rDNA sequences $(1,476$ bases) of KS-1 were aligned in comparison with available sequences from GenBank (Fig. 1). The sequences of KS-1 shared the greatest similarity with those of Marinobacter sp. R-28768 (99\% similarity; data not shown). A phylogenetic tree based on bacterial 16S rDNA sequences showed close relation-

Table 1. Biological and physiological characteristics of the isolate KS-1

\begin{tabular}{lclc}
\hline Test & Result & Test & Result \\
\hline Gram staining & - & Glucose & + \\
Motility & + & Lactose & - \\
Indole & - & Sucrose & + \\
MR & - & Mannitol & - \\
VP & - & Ducitol & - \\
Oxidase & + & Solicin & - \\
Catalase & + & Adonitol & - \\
Citrate & - & Inocitol & - \\
Gelatine & - & Sorbitol & - \\
Dnase & - & Arabinose & - \\
KIA & KIA & Raffinose & - \\
Arginine & + & Growth at & \\
Lysine & - & $37^{\circ} \mathrm{C}$ & + \\
Orinithine & - & $41^{\circ} \mathrm{C}$ & - \\
Maltose & + & $\mathrm{Growth}$ at & \\
Xylose & - & $0 \% \mathrm{NaCl}$ & - \\
Trehalose & - & $2 \% \mathrm{NaCl}$ & + \\
Cellobiose & - & $5 \% \mathrm{NaCl}$ & +
\end{tabular}

ships of KS-1 with the genera Marinobacter (Fig. 2).
Based on these results, the isolated KS-1 was identified as Marinobacter sp.

\section{Identification of mucopolysaccharides using agarose-gel electrophoresis}

Agarose-gel electrophoresis is used for the identification and separation of various mucopolysaccharides, such as slow- and fast-moving heparin, heparan sulfate, and CSs (Volpi, 1993; 1999). Analysis of KS-1 by this technique indicated the presence of a mucopolysaccharide similar to CSs (Fig. 3). However, no other mucopolysaccharides, including heparin, heparan sulfate, hyaluronic acid, or CS B, were found (Fig. 3). In addition, the gel profiles indicated that the molecular mass of the CSlike mucopolysaccharide produced by KS-1 strain is larger than those of the standard CSs (CS A, CS B, and CS C).

\section{FT-IR analysis of the CS-like mucopolysaccharide produced by Marinobacter sp. KS-1}

To determine the chemical structure of the mucopolysaccharide produced by KS-1, the molecule was analyzed by FT-IR spectrophotometry in comparison with the standard CSs. The results of the FT-IR analysis are shown in Fig. 4. Comparing the peak absorption wavelengths of the mucopolysaccharides from KS-1 and standard CSs, the FT-IR analysis revealed that the structure of the mucopolysaccharide produced by Marinobacter sp. KS-1 is similar to that of CS B, which is known to be a bioactive compound. Therefore, we conclude that the Marinobacter sp. KS-1 strain reported here produces a CS like-mucopolysaccharide that differs slightly from CS B in molecular mass.

\section{Increasing mucopolysaccharide content with longer treatment times with $1 \%$ phenol}

To obtain mucopolysaccharides, organic solvents are usually used to precipitate and remove protein (Tandavanitj et al., 1989). The yield of mucopolysaccharide increases as the time of treatment with phenol is extended. Here, the effect of the phenol treatment time on the yield of mucopolysaccharide from KS-1 pellets was investigated. To measure the mucopolysaccharide content, mucopolysaccharide was extracted from Marinobacter sp. KS-1 as described in the Materials and Methods. The content was measured in accordance with the Health Functional Food Code (KFDA; Korea Food and Drug Administration, 2009). As expected, the yield of CSlike mucopolysaccharide increased as the treatment 


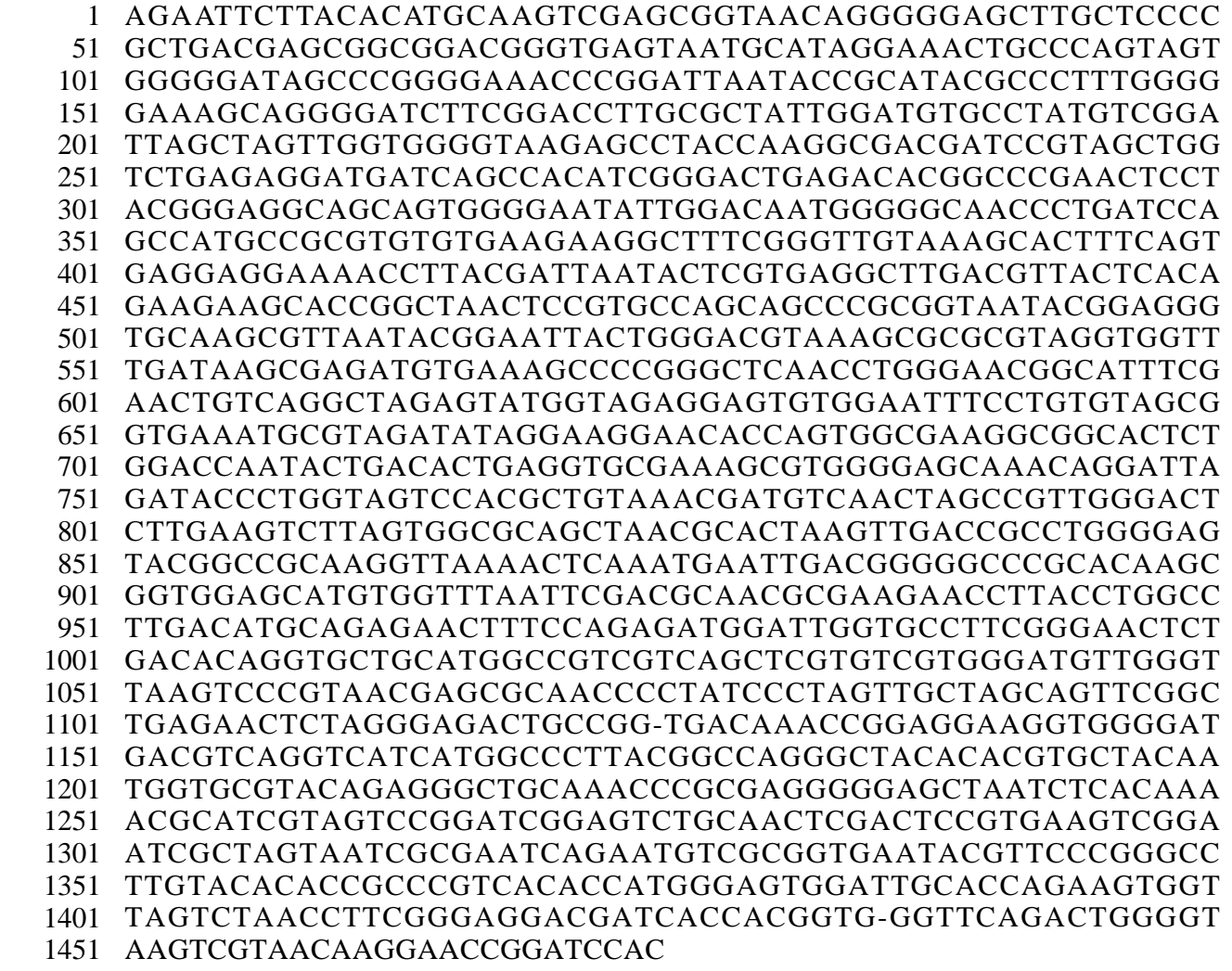

\begin{tabular}{lc}
\hline Reference (accession no.) & Identity (\%) \\
\hline Marinobacter sp. R-28768 (AM944524.1) & 99 \\
Marinobacter sp. R-28770 (AM944523.1) & 99 \\
Marinobacter sp. ASs2019 (DQ665806.1) & 99 \\
Marinobacter sp. SCSWC22 (FJ461447.1) & 99 \\
\hline
\end{tabular}

Fig. 1. 16S rDNA sequences (1476 bp) of strain KS-1 and homology search based on 16S rDNA sequences.

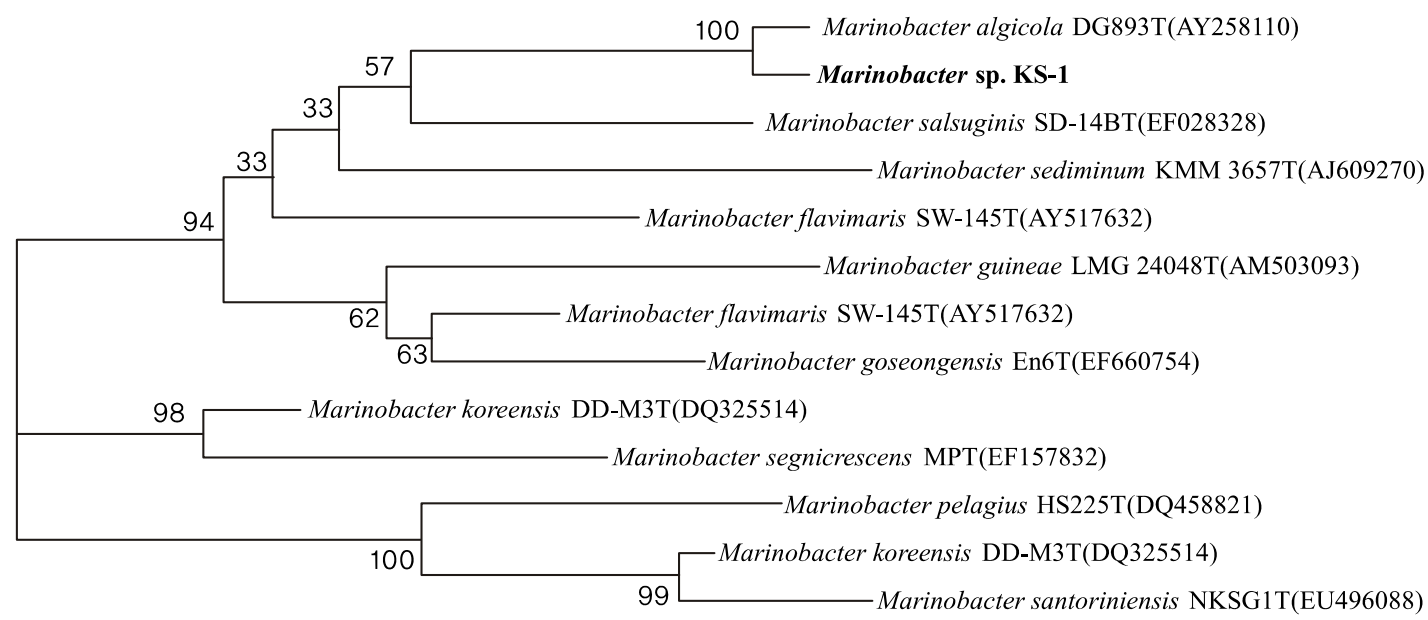

0.005

Fig. 2. Phylogenetic tree based on 16S rDNA sequences of Marinobacter sp. KS-1 and closely related members of the genus Marinobacter. Numbers at nodes are levels of bootstrap support based on neighbour-joining analyses of 1000 replications. 
Table 2. Effect of phenol treatment time on the yield of chondroitin (CS) like mucopolysaccharide

\begin{tabular}{cccc}
\hline Treatment time of $1 \%$ phenol & Wet weight of cell pellets $(\mathrm{g})$ & Total polysaccharide mass $(\mathrm{mg})$ & $\begin{array}{c}\text { CS-like mucopolysaccharide } \\
\text { content (\%) }\end{array}$ \\
\hline $15 \mathrm{~h}$ & 3 & 137.5 & 20.7 \\
$18 \mathrm{~h}$ & 3 & 157.2 & 22.1 \\
$21 \mathrm{~h}$ & 3 & 185 & 26.0 \\
\hline
\end{tabular}

The CS content was measured according to the method of Tandavanitj et al. (1989).

A

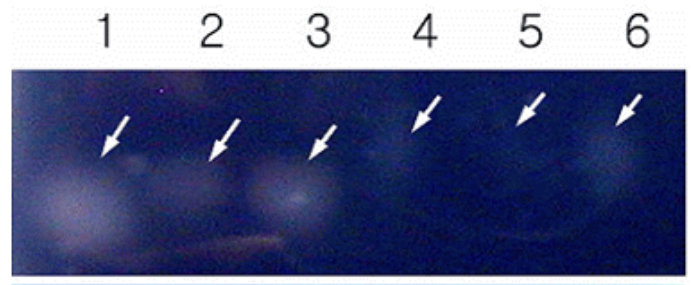

$\mathrm{B}$

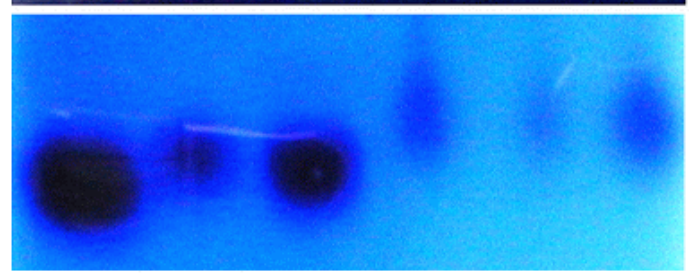

Fig. 3. Agarose-gel electrophoresis. A, Precipitation of bands in cetyltrimethyammonium bromide for $6 \mathrm{~h}$; $\mathrm{B}$, Bands stained with toluidine blue. Lane 1, CS A; lane 2, CS B; lane 3, CS C; lane 4-6, extraction of mucopolysaccharides produced by Marinobacter KS-1.

time with $1 \%$ phenol was extended (Table 2). After 15,18 , and $21 \mathrm{~h}$ of treatment using $3 \mathrm{~g}$ of harvested cell pellet, the yields of CS-like mucopolysaccharide were $20.7 \%, 22.1 \%$, and $26.0 \%$, respectively (Table $2)$. That is, the content of the CS-like mucopolysaccharide increased with the increasing treatment time of $1 \%$ phenol.

Im et al. (2009) reported that the yields of CS from various marine organisms ranged from $1.3 \%$ to $12.5 \%$. CS from shark cartilage and skate cartilage exhibited relatively high yield $(9.7 \%$ and $12.5 \%$, respectively). In comparison, the CS-like mucopolysaccharide from KS-1 showed higher efficiency in terms of yield. However, the content of the CS-like mucopolysaccharide from the microorganism in the overall specimen was lower than that from animal or marine sources.

In the current study, we isolated a marine bacterium that produces a CS-like mucopolysaccharide, and defined the characteristics of the mucopolysaccharide produced by the isolated strain. We hope that the current study will contribute to future studies on CS production to replace that from shark cartilage, a main commercial CS source.

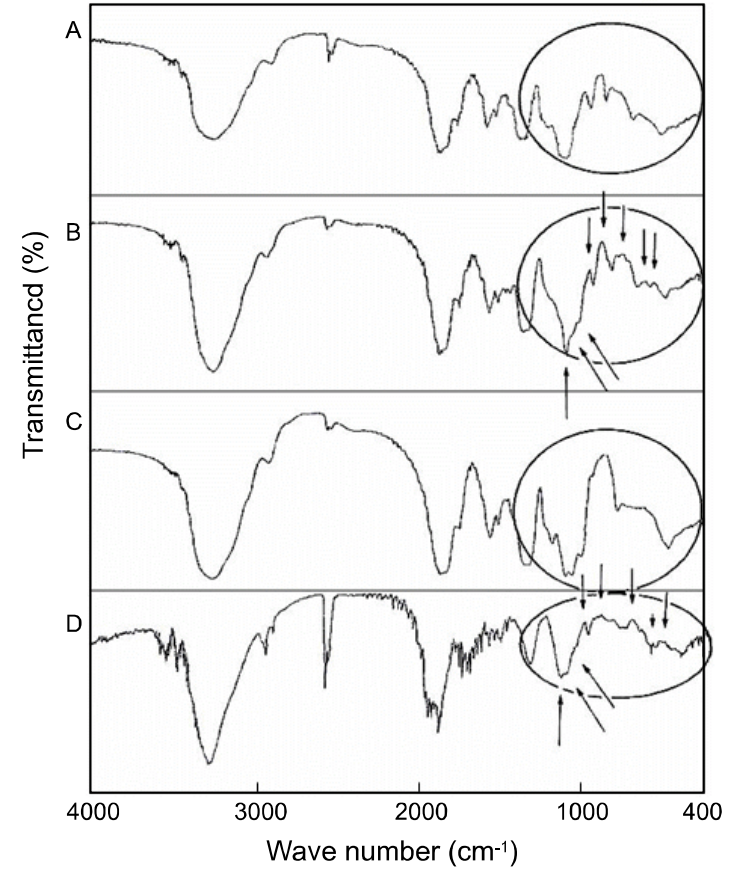

Fig. 4. Fourier transform infrared spectroscopy (FTIR) spectrums. (A) CS A; (B) CS B; (C) CS C; (D) mucopolysaccharides produced by Marinobacter KS-1.

\section{Acknowledgments}

This research was supported by the special fund of Pukyong National University donated by the SKS Trading Co. in Lynnwood, Washington, U.S.A. in memory of late Mr. Young Hwan Kang who had a deep concerns and inspiration in fisheries science.

\section{References}

Bjornsson TD, Nash PV and Schate R. 1982. The anticoagulant effect of chondroitin-4-sulfate. Thromb Res 7, 15-21.

Fontenele JB, Viana GS, Xavier-Filho J and De-Alencar JW. 1996. Anti-inflammatory and analgesic activity of a water-soluble fraction from shark cartilage. Braz J Med Biol Res 29, 643-646.

Ha BJ and Kim MH. 1999. Effect of chondroitin sulfate on 
collagen maturity and aging. J Fd Hyg Safety 14, 45-54.

Hjerpe A, Antonopoulos CA and Engfeldt B. 1979.

Determination of sulphated disaccharides from chondroitin sulfates by high-performance liquid chromatography. J Chromatogr 171, 339-344.

Im AR, Sim JS, Park Y, Hahn BS, Toida T and Kim YS. 2009. Isolation and Characterization of Chondroitin Sulfates from the By products of Marine Organisms. Food Sci Biotechnol 18, 872-877.

Kang SH, Shin H, Chang SK and Yoon HJ. 1994. Determination of sodium chondroitin sulfate by enzymatic digestion and HPLC. J Korean Soc Analytical Sci 7, 245-251.

KFDA (Korean Food and Drug Administration). 2009. The Health Functional Food Code. Seoul, Korea, III.3.4. 6.

Kim HJ, Yoon MS, Park KH, Shin JH, Heu MS and Kim JS. 2010. Processing optimization of gelatin from rockfish skin based on yield. Fish Aqua Sci 13, 1-11.

Krueger RCJ, Hennig AK and Schwartz NB. 1992. Two immunologically and developmentally distinct chondroitin sulfate proteoglycans in embryonic chick brain. J Biol Chem 267, 12149-12161.

Mac-Rae SM, Edelhauser HF, Hyndiuk RA, Burd EM and Schultz RO. 1983. The effects of sodium hyaluronate, chondroitin sulfate, and methycellulose on the corneal endothelium and intraocular pressure. Am J Ophthalmol 95, 332-341.

Moon JH, Ryu HS, Yang HS and Suh JS. 1998. Antimutagenic and anticancer effects of glycoprotein and chondroitin sulfates from sea cucumber (Stichopus japonicus). J Korean Soc Food Sci Nutr 27, 350-358.

Mourao PA, Marians SP and Mauro SGP. 1996. Structure and anticoagulant activity of a fucosylated chondroitin sulfate from echinoderm. J Biol Chem 271, 2397323984.

Moyer CL, Dobbs FC and Karl DM. 1994. Estimation of diversity and community structure through RELF distribution analysis of bacterial 16S-rRNA genes from a microbial mat at an active, hydrothermalvent, Loihi Seamount, Hawii. Appl Env Microbiol 60, 871-879.

Prince CW and Navia JM. 1983. Glycosaminoglycan alterations in rat bone due to growth and fluorosis. J Nutr 113, 1576-1582.

Sim JS, Jun GJ, Toida T, Cho SY, Choi DW, Chang SY, Linhardt RJ and Kim YS. 2005. Quantitative analysis of chondroitin sulfate in raw materials, ophthalmic solutions, soft capsules, and liquid preparations. J Chromatogr B 818, 133-139.

Smibert RM and Krieg N R. 1994. Phenotypic characterization. In Methods for General and Molecular Bacteriology. Gerhardt P, Murray RGE, Wood WA and Krieg NR, ed. ASM Press, Washington DC, U.S.A., 607-654.

Smith RL and Schurman DJ. 1983. Comparison of cartilage destruction between infectious and adjuvant arthritis. J Orthop Res, 1, 136-143.

Taga N. 1968. Some ecological aspects of marine bacteria in the Kuroshio current. Bull Misaki Mar Biol Kyoto Univ, 12, 50-76

Tanaka K. 1978. Physicochemical properties of chondroitin sulfate. I. Ion binding and secondary structure. J Biochem 83, 647-653.

Tandavanitj S, Ishida S and Okutani K. 1989. Isolation and characterization of an extracellular mucopolysaccharide produced by a marine strain of Pseudomonas. Nippon Suisan Gakkaishi 55, 2015-2019.

Volpi N. 1993. "Fast moving" and "slow moving” heparins, dermatan sulfate, and chondroitin sulfate: qualitative and quantitative analysis by agarose-gel electrophoresis. Carbohydr Res 247, 263-278.

Volpi N. 1996. Electrophoresis separation of glycosaminoglycans on nitrocellulose membranes. Anal Biochem 240, 114-118.

(Received 16 June 2010; Revised 21 July 2010; Accepted 10 September 2010) 